\title{
Condições de Saúde bucal e acuidade visual dos estudantes em um município do Baixo Amazonas
}

\author{
Oral health and visual acuity conditions of students in a municipality of Baixo Amazonas \\ Condiciones de salud bucal y agudeza visual de estudiantes en una ciudad de Baixo Amazonas
}

Lauramaris de Arruda Regis-Aranha ${ }^{1}$ (1) Marcelo de Castro Meneghim² (1) Ângelo Esmael da Silva Maklouf ${ }^{3}$ (D) Guilherme Regis Aranha ${ }^{4}$ (1) Adriana Beatriz Silveira Pinto ${ }^{1}$ (C) Shirley Maria de Araújo Passos ${ }^{1}$ (D) Ângela Xavier Monteiro ${ }^{1}$ (1)

1.Universidade do Estado do Amazonas. Manaus, AM, Brasil.

2.Universidade Estadual de Campinas, Faculdade de Odontologia de Piracicaba. Piracicaba, SP, Brasil.

3. Secretaria Municipal de Saúde. Barreirinha, AM, Brasil.

4. Universidade de Santo Amaro. São Paulo, SP, Brasil.

\section{Resumo}

Objetivo: Avaliar a prevalência da cárie dentária e da deficiência visual nos escolares do ensino fundamental da rede pública de ensino de Barreirinha-AM e verificar os fatores associados a estas condições. Método: Estudo transversal, com 1.828 estudantes entre 06 e 17 anos, da rede pública de ensino, Barreirinha. A avaliação da cárie dentária seguiu as recomendações da Organização Mundial da Saúde utilizando os critérios estabelecidos para as condições dentárias, tanto para dentes decíduos, quanto para permanentes: cariados, perdidos e obturados. O índice Significant Caries Index mediu a severidade da cárie dentária nos indivíduos mais afetados com a doença. Realizou-se avaliação para acuidade visual e analisou-se idade, sexo e escola. Resultados: Evidenciou-se elevada prevalência de cárie dentária, desigualdade em sua distribuição e associação entre esta doença e sexo $(p=0,005)$. A prevalência de dificuldades visuais entre os escolares foi baixa. Conclusão e implicações para prática: $O$ estudo sugere carência de tratamento odontológico restaurador e elevada prevalência de cárie dentária, evidenciando a necessidade de planejamento e desenvolvimento de ações de promoção de saúde bucal, com o intuito de minimizar os agravos provocados pela doença.

Palavras-chave: Acuidade Visual; Saúde Bucal; Índice CPO; Estudantes; Prevalência.

\section{Abstract}

Objective: To evaluate the prevalence of dental caries and visual acuity deficiency in elementary school students of the public school system in the municipality of Barreirinha- AM and check the factors associated with these conditions. Method: Crosssectional study with 1,828 students aged 6 to 17 in the public school system of the municipality of Barreirinha-AM. The dental cavity evaluation followed the recommendations of the World Health Organization using the established criteria for dental conditions, for deciduous as for permanent teeth: decayed, missing and filled. The Significant Caries Index was used to measure the denta caries severity in the individuals most affected by this disease. Visual acuity was assessed and age, sex and school were analyzed. Results: It became evident high prevalence of dental cavities, inequality in its distribution, and relation between this disease and gender $(p=0.005)$. The visual deficit frequency was low among the students. Conclusion and implications for practice: The study indicates a lack of dental restoration treatment and high dental cavity prevalence, highlighting the need for planning and development of oral health promotion actions, with the purpose of minimizing the aggravations caused by the disease.

Keywords: Visual Acuity; Oral Health; DMF Index; Students; Prevalence.

\section{Resumen}

Objetivo: Evaluar la prevalencia de caries dentales y discapacidad visual en estudiantes de la enseñanza primaria del sistema escolar público de Barreirinha-AM, y verificar los factores asociados con estas condiciones. Método: Estudio transversal realizado con 1.828 estudiantes entre 06 y 17 años, de escuelas públicas de Barreirinha-AM. La evaluación de las caries dentales siguió las recomendaciones de la Organización Mundial de la Salud, utilizando los criterios establecidos para las condiciones dentales tanto para dientes temporales como permanentes: cariados, perdidos y obturados. El índice Significant Caries Index midió la gravedad de la carie dental en las personas más afectadas por la enfermedad. Se realizó la evaluación para agudeza visual y se analizó edad, sexo y nivel educativo. Resultados: Hubo alta prevalencia de carie dental, desigualdad en su distribución y asociación entre esta enfermedad y el sexo $(p=0,005)$. La prevalencia de dificultades visuales entre los estudiantes fue baja. Conclusión e implicaciones para la práctica: El estudio sugiere una carencia de tratamiento dental restaurador y una alta prevalencia de caries dentales, evidenciando la necesidad de planificación y desarrollo de acciones de promoción a la salud bucal, con el fin de minimizar los problemas ocasionados por la enfermedad.

Palabras clave: Agudeza Visual; Salud Bucal; Índice CPO; Estudiantes; Prevalencia.
Autor correspondente:

Lauramaris de Arruda Regis-Aranha.

E-mail: laranha@uea.edu.br.

Recebido em 17/07/2020.

Aprovado em 15/12/2020.

DOl:https://doi.org/10.1590/2177-9465-EAN-2020-0244 


\section{INTRODUÇÃO}

Na perspectiva de ampliar as ações de prevenção, promoção e atenção à saúde dos alunos da rede pública de ensino, foi instituído o Programa Saúde na Escola (PSE), em 2007. O PSE determina que as equipes de Saúde da Família devem constituir, junto com a Educação Básica, estratégias para a integração e articulação permanente entre as políticas e ações de educação e saúde, com a participação da comunidade escolar. Sabe-se que a escola é espaço de grande relevância para o desenvolvimento de um programa de educação para a saúde de crianças e adolescentes. O Ministério da Saúde, por meio do PSE, possui inúmeras ações, sendo algumas delas já bem definidas e, dentre essas últimas, encontram-se a saúde bucal e a saúde ocular, que podem ser consideradas importantes meios de vigilância em saúde dessa população. ${ }^{1}$

Nesse sentido, o Ministério da Saúde vem intensificando sua atuação em ações específicas para a população infantil. Em 2015, foi instituída a Política Nacional de Atenção Integral à Saúde da Criança (PNAISC). Suas ações se organizam a partir das Redes de Atenção à Saúde (RAS), com ênfase na Rede de Atenção à Saúde Materna e Infantil, tendo a Atenção Básica à Saúde (ABS) como ordenadora das ações, com estratégias direcionadas à criança, garantindo o direito à vida e à saúde, o acesso universal de todas as crianças à saúde, à equidade, à integralidade do cuidado, à humanização da atenção e à gestão participativa. $^{2}$

Com base em uma visão ampliada da saúde da comunidade e das famílias, é muito importante que os profissionais que compõem as equipes de saúde trabalhem interprofissionalmente, contribuindo para a melhoria na qualidade de vida. ${ }^{3}$ Ações de promoção e prevenção da saúde bucal e saúde ocular contribuem positivamente e auxiliam na minimização dos impactos negativos que tais problemas têm na vida das pessoas. ${ }^{4,5}$

A cárie dentária constitui um dos principais agravos enfrentados pela Odontologia ${ }^{4}$ e é considerada prioridade em saúde pública. A doença dentária, uma vez não tratada, pode levar à dor, além de dificuldades na mastigação, em comer, em sorrir, como também ao absenteísmo no trabalho e na escola. ${ }^{6}$ No Brasil, os quatro grandes levantamentos com amplitude nacional, realizados em 1986, 1996, 2003 e 2010, evidenciaram grande declínio na prevalência da cárie dentária. ${ }^{7}$ No entanto, quanto à sua distribuição, estudos evidenciam grandes desigualdades regionais, afetando desproporcionalmente grupos mais pobres e marginalizados da sociedade, ${ }^{6,8-13}$ tornando-os suscetíveis à concentração de níveis mais elevados dessa doença. ${ }^{6-8,10,14}$

Com efeito, é fundamental conhecer a realidade das condições de saúde bucal da população, ${ }^{15}$ pois as análises epidemiológicas acerca da cárie dentária permitem identificar se as ações realizadas foram efetivas para sua redução, ${ }^{12}$ como também identificar grupos que concentram maior prevalência dessa doença, tornando possível a visualização dos grupos com menor acesso a cuidados restauradores. ${ }^{15}$ Assim sendo, com a utilização do índice Significant Caries Index ( $\mathrm{SiC}$ ), é possível obter o impacto da cárie dentária nos grupos que apresentam maior prevalência da doença. ${ }^{16}$

O Ministério da Saúde e o Ministério da Educação, em parceria, instituíram em 2007 o Projeto "Olhar Brasil". O "Olhar Brasil" tem como objetivo identificar e corrigir problemas de visão em alunos matriculados na rede pública de ensino da Educação Básica, priorizando, inicialmente, o atendimento ao ensino fundamental, em alfabetizados cadastrados no "Programa Brasil Alfabetizado" e na população com idade igual ou acima de 60 anos. Essa iniciativa, além de ampliar o acesso à saúde ocular, tem como objetivo melhorar o aprendizado de crianças e adultos em fase escolar. ${ }^{17}$

A visão é fundamental para a relação do indivíduo com o meio ao seu redor, pois interfere no seu desenvolvimento intelectivo e interpessoal, ${ }^{18,19}$ acometendo $18,60 \%$ da população brasileira. ${ }^{20}$ Estudos apontam que escolares com distúrbios oculares podem apresentar dor de cabeça, olhos vermelhos, cansaço visual e tonturas, ocorrências que interferem negativamente na evolução da aprendizagem e, muitas vezes, resultam na evasão escolar. 1,17,20

Diante do exposto, a realização de testes de triagem de acuidade visual, bem como o encaminhamento para possíveis tratamentos, é fundamental para detecção e intervenção precoce nessas alterações, minimizando os danos causados e prevenindo agravos mais complexos. .,9 $^{2}$

Considerando inexistir estudos anteriores acerca das informações epidemiológicas quanto à distribuição da cárie dentária e do déficit visual no município de Barreirinha-AM, este estudo teve como objetivo avaliar a prevalência da cárie dentária e da deficiência visual nos escolares do ensino fundamental da rede pública deste município e verificar os fatores associados a estas condições.

\section{MÉTODOS}

O estudo foi realizado no município de Barreirinha, região do Baixo Amazonas, distante $326 \mathrm{~km}$ em linha reta de Manaus (Capital). O seu acesso se dá por meio de transporte fluvial ou aéreo e, segundo o Instituto Brasileiro de Geografia e Estatística (IBGE), contava com 27.355 habitantes no censo de 2010, sendo $45,4 \%$ dessa população residente na área urbana. Possui um total de treze distritos, contando com Barreirinha, a sede. A rede de assistência à saúde da sede conta com um hospital geral e quatro Unidades Básicas de Saúde (UBS), totalizando quatro Equipes de Saúde Bucal (ESB). Somente seis distritos possuem UBS e destes, apenas quatro possuem ESB. Contase, também, com uma UBS Fluvial, sendo composta, quanto à área da odontologia, por uma ESB. Estima-se que a cobertura populacional de ESB na ABS foi de apenas $34,81 \%$ no município de Barreirinha, em 2014. ${ }^{21,22}$

Este é um estudo transversal e observacional, iniciado em agosto de 2014 e finalizado em dezembro de 2017. Optou-se por avaliar alunos de ambos os sexos, entre 6 e 17 anos, matriculados no ensino fundamental de escolas municipais e estaduais na cidade de Barreirinha (área urbana), Amazonas, quanto à prevalência dos números de dentes cariados, perdidos e obturados, para 
dentição permanente (CPO-D), dos números de dentes cariados, perdidos e obturados, para dentição decídua (ceo-d) como também a deficiência visual e verificar os fatores associados a estas condições. Segundo informações das Secretarias de Educação: Municipal e Estadual, em 2014, a sede do município (área urbana) contava com um total de 5 escolas públicas no Ensino Fundamental (03 municipais e 02 estaduais), perfazendo um total de 2.807 estudantes (1.325 e 1.482 estudantes da rede municipal e estadual de ensino, respectivamente). Por se tratar de um município que não apresenta escolas particulares, como também Índice de Desenvolvimento Humano (IDH) de 0,574, considerado baixo, optou-se por não coletar informações socioeconômicas. ${ }^{23}$

Por se tratar de um estudo de conveniência, a preferência pelo município do estudo se deu por sua participação no campo de estágio da disciplina de Estágio Rural em Saúde Coletiva da Universidade do Estado do Amazonas. A referida disciplina é obrigatória e envolve acadêmicos do último período dos cursos de Medicina, Odontologia e Enfermagem que, no momento realizam atividades na $A B S$ de alguns municípios situados no interior do estado do Amazonas.

Este estudo foi aprovado pelo Comitê de Ética em Pesquisa da Escola Superior de Ciências da Saúde/Universidade do Estado do Amazonas (CAAE: 31319614.8.0000.5016). Foram entregues aos 2.807 estudantes o Termo de Consentimento Livre e Esclarecido (TCLE) e cada escolar foi convidado a participar do estudo mediante suas livres escolhas e atendendo aos princípios éticos. Somente alunos que concordaram em participar da pesquisa e cujos pais assinaram o TCLE foram avaliados quanto à saúde bucal e acuidade visual. Foram excluídos estudantes que usavam aparelho ortodôntico fixo, portadores de completa deficiência visual (cegos), que possuíam idade superior a 17 anos e aqueles que não concordaram em realizar o exame.

Sendo assim, foram assinados pelos pais, um total de 1.859 TCLE. Desses, 1.828 estudantes estavam dentro dos critérios de inclusão. No entanto, a amostra final de conveniência foi composta por 1.828 escolares com idade entre 6 e 17 anos, os quais concordaram em participar do exame para cárie dentária, representando uma taxa de resposta de $80,8 \%$ dos alunos da rede municipal e 51,1\% dos matriculados na rede estadual. Quanto à acuidade visual, somente 1.407 estudantes com idade entre 6 e 17 anos concordaram em participar, representando uma taxa de resposta de $47,1 \%$ dos alunos da rede municipal e $52,9 \%$ dos matriculados na rede estadual. As perdas se deram pela dificuldade em contatar os pais, recusa dos responsáveis em assinar a autorização, como também pelo absenteísmo dos alunos no momento do exame.

\section{Levantamento Epidemiológico da Cárie dentária}

A avaliação da saúde bucal foi realizada por meio de exame epidemiológico nas escolas, sob a luz natural e seguindo a padronização da Organização Mundial de Saúde (OMS). ${ }^{24}$ Para avaliação da saúde bucal das crianças (6 a 11 anos), foi utilizado o índice ceo-d obtido pela soma de dentes decíduos cariados, extraídos ou com indicação de extração por lesão de cárie e obturados. Em relação aos adolescentes (12 a 17 anos), foi utilizado o índice CPO-D obtido pela soma de dentes permanentes cariados, perdidos e obturados. Foi utilizado também o registro das necessidades de tratamentos nos dentes decíduos e permanentes. ${ }^{24}$

Para facilitar a comparação entre dentição decídua e dentição permanente, optou-se pela separação das faixas etárias de 6 a 11 anos (crianças) e de 12 a 17 anos (adolescentes).

O índice SiC foi utilizado para avaliar a severidade da cárie dentária no terço do grupo que apresentou maior experiência da doença. Para a análise, dividiu-se em dois grupos: o primeiro com o terço dos indivíduos com os maiores índices de cárie (grupo $\mathrm{SiC}$, alta experiência de cárie) e o segundo com os demais indivíduos com os menores índices (baixa experiência de cárie). ${ }^{12}$

Para a coleta de dados, um grupo de examinadores, acadêmicos do último período do curso de Odontologia, foi treinado previamente, quanto à padronização de técnicas, por uma professora com expertise na área e que integrou o grupo da pesquisa. $O$ treinamento para cada equipe avaliadora seguiu as recomendações da OMS, compreendendo aula teórica (4 horas) e treinamento prático (8 horas). O valor de Kappa para concordância intraexaminador e interexaminador para cárie dentária variou de 0,70 a 0,89 e de 0,70 a 0,86, respectivamente.

\section{Levantamento Epidemiológico da Acuidade Visual}

Para avaliação da acuidade visual, seguindo a padronização do Ministério da Saúde, ${ }^{1}$ foi utilizada a Escala de Snellen na própria escola, instalada em ambiente calmo, com boa iluminação (a luz atrás do escolar), retirados os objetos dispersivos que estivessem no campo de visão, cartaz com a Escala de Snellen posicionado em uma parede a uma distância de 5 metros de modo que a linha de acuidade visual 0,8 a 1,0 ficasse na altura dos olhos do examinado. ${ }^{1,17}$

Como variáveis de interesse, foram avaliados: idade, sexo, tipo de escola e cárie dentária. A partir da variável idade, foi empregada uma nova variável chamada faixa etária com intervalos de aproximadamente 5 e 6 anos (6-11 e 12-17), com o objetivo de caracterizar os grupos de crianças e adolescentes, respectivamente.

Durante o exame, observou-se a presença ou ausência do uso de óculos, como também sinais e/ou sintomas indicadores de possíveis problemas visuais como: ardência, lacrimejamento, olho vermelho, secreção, atos de apertar ou arregalar os olhos para enxergar melhor, inclinação de cabeça, visão embaçada, dor de cabeça e estrabismo. ${ }^{17}$

O processo de treinamento do grupo de examinadores, acadêmicos do último período dos cursos de Medicina e Enfermagem, foi realizado pela mesma professora com expertise na área. Compreendeu uma carga horária total de 16 horas (teoria e prática), a fim de padronizar a técnica no mesmo grupo de avaliadores, que realizaram tanto a avaliação da cárie dentária quanto da acuidade visual. Como unidade de medida, os valores foram de 0,1 a 1,0 . Sendo classificados, como tendo 
Acuidade Visual (AV) normal, escolares que apresentaram AV maior ou igual a 0,8 em ambos os olhos para todas as idades e estabelecendo-se como déficit de AV valores menores ou iguais a 0,7 em um ou ambos os olhos, seguindo a padronização do Ministério da Saúde. ${ }^{1}$

\section{Análise estatística}

Os dados foram apresentados por meio de tabelas, onde se calcularam as frequências absolutas, simples e relativas para os dados categóricos. $\mathrm{Na}$ análise dos dados quantitativos, quando aceita a hipótese de normalidade por meio do teste de ShapiroWilk, foi calculada a média, o desvio-padrão (Dp) e aplicados os testes paramétricos $t$-student e Análise de Variância - ANOVA. Já no caso da rejeição da hipótese de normalidade, optou-se por calcular a mediana, os quartis $\left(Q_{i}\right)$ e aplicar os testes não paramétricos de Mann-Whitney e Kruskal-Waliis.

A análise univariada foi desempenhada com o objetivo de observar associação entre as variáveis dependentes (CPO-D, ceo-d e AV) e as variáveis independentes (idade, sexo e escola), utilizando os testes qui-quadrado de Pearson e Teste $t$-student.

O software utilizado na análise dos dados foi o programa Epi Info, versão 7.2, distribuído gratuitamente pelo Centro de Controle e Prevenção de Doenças Norte-americano - CDC. O nível de significância para a aplicação dos testes estatísticos foi de $5 \%$.

\section{RESULTADOS}

\section{Cárie dentária}

Participaram do estudo para cárie dentária 1.828 estudantes, dos quais $957(52,4 \%)$ pertenciam ao sexo feminino. A prevalência encontrada foi de $67,2 \%$ (736 alunos) daqueles com idade entre 6 e 11 anos e de $75,8 \%$ (556 alunos) daqueles com idade entre 12 e 17 anos.

O valor do índice ceo-d foi de 2,65 (DP =2,82), SiC de 6,04 e no grupo com baixa experiência de cárie foi de 0,96, evidenciando desigualdade na distribuição da doença no local estudado. Encontrou-se associação significativa no grupo do sexo masculino com aqueles que apresentavam piores ceo-d e piores dos componentes cariado e extraído ou com indicação de extração ( $p=0,005 ; p=0,027 ; p=0,003)$. Aos 6 anos de idade, $30,5 \%$ dos escolares estavam livres de cárie (ceo=0) (Tabela 1).

O valor do índice CPO-D foi de 2,65 (DP = 2,64), SiC de 5,58 e no grupo com baixa experiência de cárie foi de 1,19, evidenciando desigualdade na distribuição da doença no local da pesquisa. Aos 12 anos de idade, o índice CPO-D foi de 1,94 $(D P=2,19)$. Não se encontrou associação significativa entre sexo com aqueles que apresentavam piores CPO-D e piores dos componentes cariado, extraído ou com indicação de extração e obturado (Tabela 2).

A necessidade de tratamento odontológico encontrada foi de $72,9 \%$ (798 alunos) daqueles com idade entre 6 e 11 anos e de $64,8 \%$ (475 alunos) daqueles com idade entre 12 e 17 anos, sendo a necessidade de restauração em uma ou mais superfície a mais evidenciada tanto na dentição decídua como na dentição permanente. No entanto, chama atenção que a segunda maior necessidade de tratamento na dentição permanente é a extração dentária (Tabela 3).

\section{Acuidade visual}

Foram submetidos ao exame de triagem 1.407 estudantes, dos quais 745 (52,9\%) pertenciam ao sexo feminino. Analisando separadamente os dois olhos, 86 estudantes $(6,1 \%)$ apresentaram AV alterada no olho direito e 94 estudantes (6,7\%) no olho esquerdo. Não houve associação estatisticamente significante ao relacionar as variáveis déficit visual para olho direito e para o olho esquerdo com as faixas etárias $(p=0,912$ e $p=0,938$, respectivamente) ao se realizar o Teste qui-quadrado de Person (Tabela 4). Ao longo do exame, notaram-se em 451 (32,1\%) escolares alguns sinais e/ou sintomas de problemas visuais.

$\mathrm{Na}$ Tabela 5, a frequência de baixa acuidade foi de $8,4 \%$ (118/1407), com IC95\% (7,0\% - 9,9\%), sendo estes encaminhados para exame médico-oftalmológico. Foi encontrada uma relação entre baixa acuidade visual e a variável tipo de escola $(p=0,097)$, porém, não estatisticamente significativa. Não se encontrou associação significativa entre baixa acuidade visual e cárie dentária.

\section{DISCUSSÃO}

A cárie dentária é considerada um dos grandes problemas de saúde pública, pois, além de causar impactos negativos à população, impõe ônus econômico capaz de exacerbar o ônus de outras doenças. ${ }^{4,6} \mathrm{~A}$ última pesquisa nacional de saúde bucal, realizada em 2010, no Brasil, evidenciou declínio da cárie dentária para todas as idades, entretanto as regiões Norte, Nordeste e Centro-Oeste do país apontaram médias mais elevadas de CPO-D. ${ }^{7}$

Chama atenção a elevada prevalência de cárie dentária encontrada neste estudo. Esses resultados podem estar relacionados à baixa cobertura ESB encontrada no município, uma vez que o trabalho em equipes multiprofissionais na Estratégia Saúde da Família (ESF), como também, ações educativas, preventivas e curativas ficam comprometidas, dificultando a integralidade da assistência à saúde e o trabalho em rede. ${ }^{2}$ Desta forma, é imprescindível a realização do monitoramento da saúde bucal, uma vez que possibilita identificar se as ações realizadas estão sendo efetivas, como também contribui para que medidas essenciais sejam traçadas. ${ }^{10-12,25}$

Salienta-se que, neste estudo, a meta da OMS para 2010 não foi atingida, que era apresentar CPO-D menor que 1,0 para a idade de 12 anos e $90 \%$ livres de cárie aos 5 ou 6 anos de idade. Estes resultados devem apoiar formuladores de política, visando diminuir a prevalência de cárie dentária e o seu impacto na idade adulta. ${ }^{10}$ É sabido que o aumento do contato da população com as mais variadas formas de flúor contribuiu para a redução da cárie dentária. ${ }^{14}$ Uma das estratégias amplamente conhecida e bem-sucedida para a prevenção da cárie dentária é a fluoretação da água de abastecimento público, razão pela qual a universalização desse acesso servirá como fator protetor 


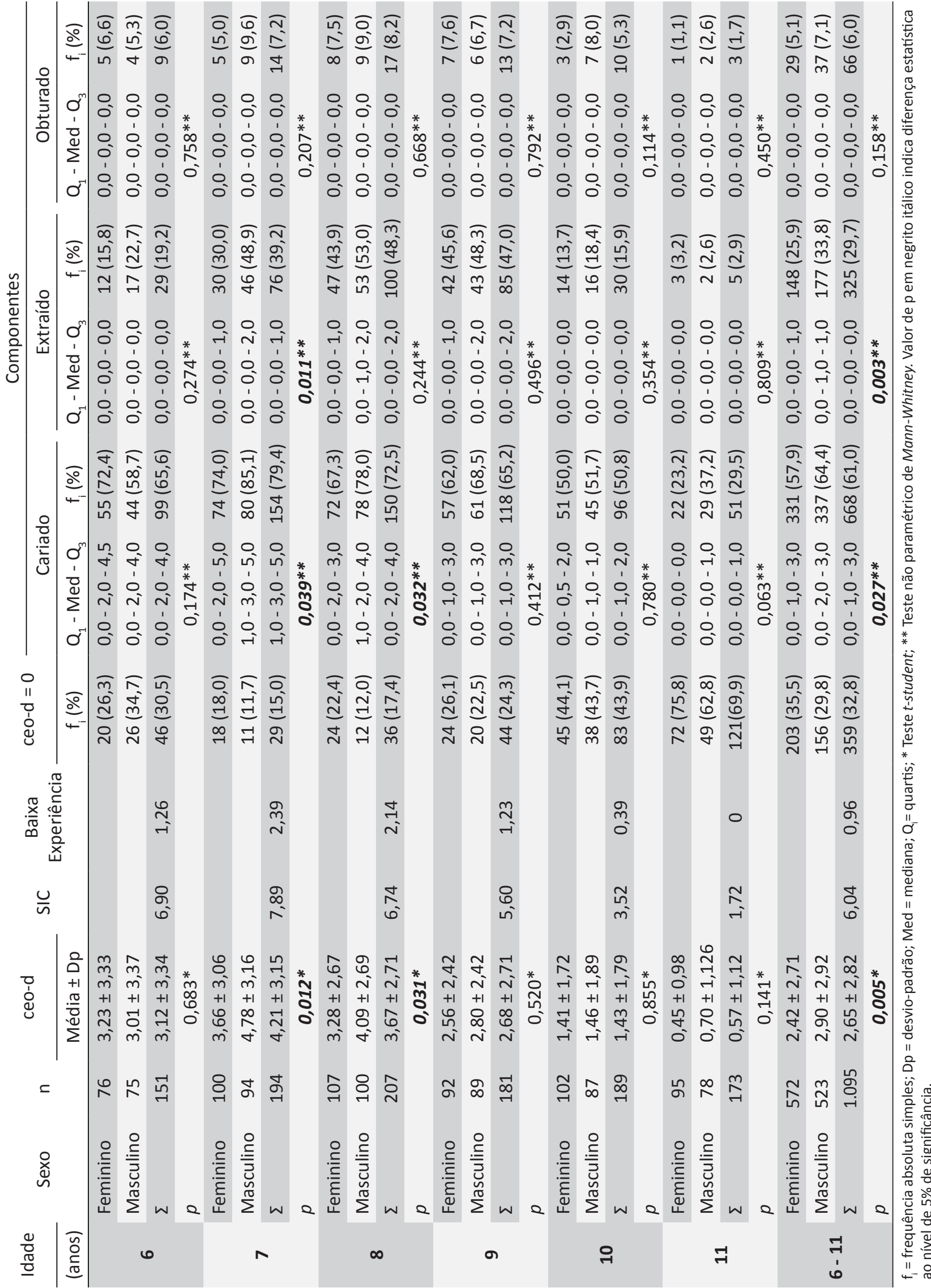









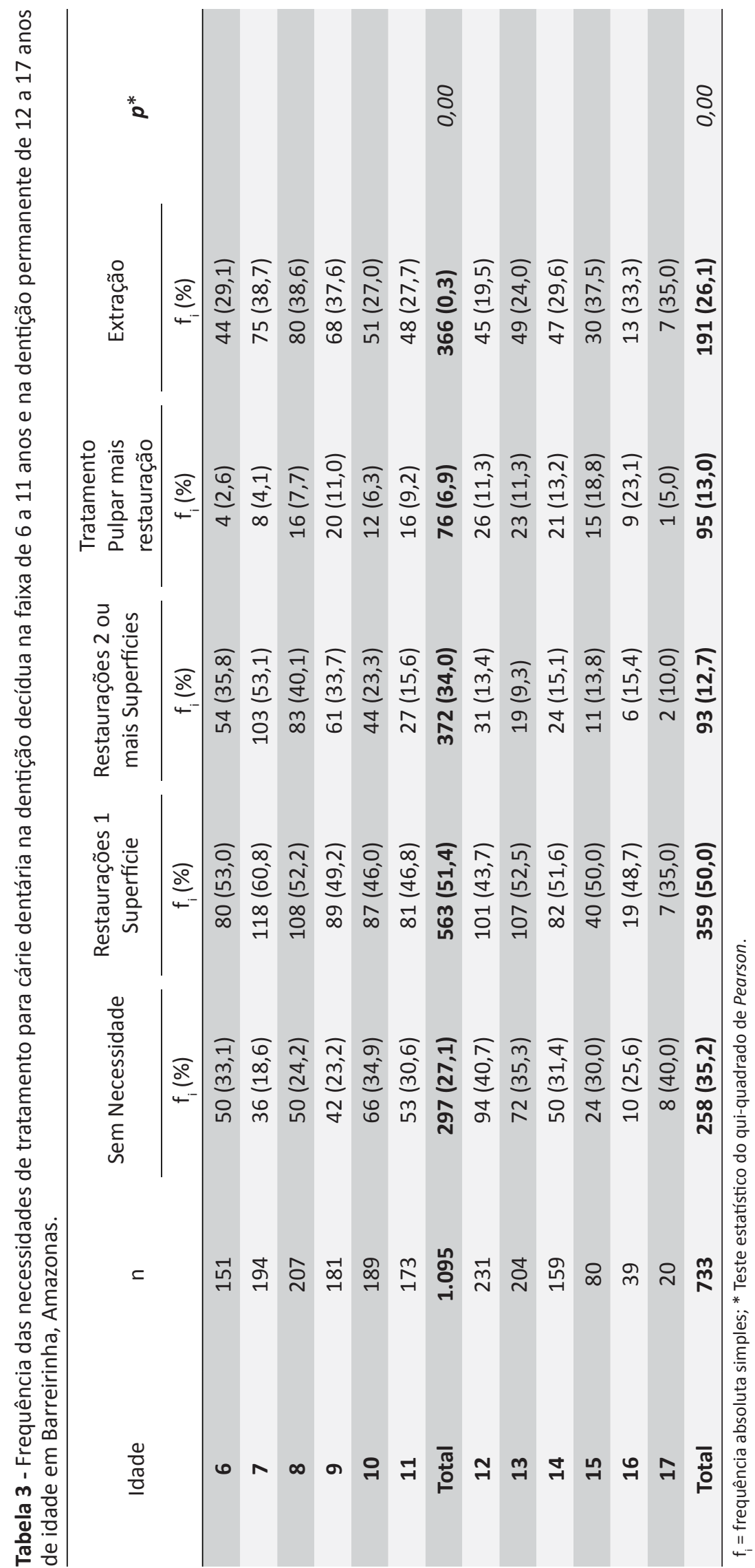


Tabela 4 - Distribuição dos escolares por idade de acordo com o Déficit Visual (olho direito e olho esquerdo) em Barreirinha, Amazonas

\begin{tabular}{|c|c|c|c|c|c|}
\hline \multirow[b]{2}{*}{ FAIXA ETARIA } & \multirow[b]{2}{*}{$\mathrm{n}$} & \multirow{2}{*}{$\begin{array}{c}\text { OLHO DIREITO } \\
<0,8 \\
\mathrm{f}_{\mathrm{i}}(\%) \\
\end{array}$} & \multirow{2}{*}{$\frac{p^{*}}{0,911}$} & \multirow{2}{*}{$\begin{array}{c}\text { OLHO ESQUERDO } \\
<0,8 \\
\mathrm{f}_{\mathrm{i}}(\%)\end{array}$} & \multirow{2}{*}{$\begin{array}{c}p^{*} \\
0,938\end{array}$} \\
\hline & & & & & \\
\hline 7 & 56 & $3(5,4)$ & & $3(5,4)$ & \\
\hline 8 & 126 & $9(7,1)$ & & $9(7,1)$ & \\
\hline 10 & 156 & $12(7,7)$ & & $11(7,1)$ & \\
\hline 11 & 164 & $5(3,1)$ & & $6(3,7)$ & \\
\hline 6 a 11 & 679 & $42(6,19 \%)$ & & $45(6,63 \%)$ & \\
\hline 12 & 229 & $17(7,4)$ & & $18(7,9)$ & \\
\hline 16 & 39 & $2(5,1)$ & & $3(7,7)$ & \\
\hline 17 & 20 & - & & - & \\
\hline 12 a 17 & 728 & $44(6,04 \%)$ & & $49(6,73 \%)$ & \\
\hline TOTAL & 1407 & $86(6,11 \%)$ & & $94(6,68 \%)$ & \\
\hline
\end{tabular}

* Teste estatístico do qui-quadrado de Pearson.

Tabela 5 - Distribuição da freqüência dos escolares quanto ao sexo, tipo de escola e idade de acordo com a Acuidade Visual e Cárie dentária em Barreirinha, Amazonas.

\begin{tabular}{|c|c|c|c|c|}
\hline \multirow[t]{2}{*}{ Variáveis } & \multirow[t]{2}{*}{$n$} & $\begin{array}{c}\text { Acuidade Visual Baixa } \\
\qquad(<0,8)\end{array}$ & $\begin{array}{c}\text { Acuidade Visual Normal } \\
\qquad(\geq 0,8)\end{array}$ & \multirow[t]{2}{*}{$p$} \\
\hline & & $\mathrm{f}_{\mathrm{i}}(\%)$ & $f_{i}(\%)$ & \\
\hline Sexo & & & & $0,288^{*}$ \\
\hline Feminino & 745 & $68(9,1)$ & $677(90,9)$ & \\
\hline Masculino & 662 & $50(7,6)$ & $612(92,4)$ & \\
\hline Total & 1.407 & $118(8,4)$ & $1289(91,6)$ & \\
\hline Escolas & & & & $0,097^{*}$ \\
\hline Estaduais & 745 & $71(9,5)$ & $673(90,5)$ & \\
\hline Municipais & 662 & $47(7,1)$ & $616(92,9)$ & \\
\hline Total & 1.407 & $118(8,4)$ & $1.289(91,6)$ & \\
\hline Idades & & & & $0,318^{* *}$ \\
\hline 6-11 anos & 679 & $57(8,4)$ & $622(91,6)$ & \\
\hline $12-17$ anos & 728 & $61(8,4)$ & $667(91,6)$ & \\
\hline Média \pm Dp & & $11,2 \pm 2,5$ & $11,4 \pm 2,5$ & \\
\hline Total & 1.407 & $118(8,4)$ & $1289(91,6)$ & \\
\hline \multicolumn{5}{|l|}{ Cárie dentária } \\
\hline ceo- $d<1$ & 262 & $18(31,6)$ & $244(39,2)$ & $0,256^{*}$ \\
\hline ceo- $d \geq 1$ & 417 & $39(68,4)$ & $378(60,8)$ & \\
\hline CPO-D < 1 & 177 & $14(23,0)$ & $163(24,4)$ & $0,796 *$ \\
\hline CPO-D $\geq 1$ & 551 & $47(77,0)$ & $504(75,6)$ & \\
\hline
\end{tabular}

$\mathrm{f}_{\mathrm{i}}=$ frequência absoluta simples; * Teste estatístico do qui-quadrado de Pearson, ** Teste t-student para comparação das médias. 
à saúde, ao mesmo tempo em que contribuirá para a redução de desigualdade.8,9,16,26,27 Entretanto, Barreirinha não possui flúor em sua água de abastecimento.

A Polarização da cárie dentária foi encontrada no presente estudo, pois uma concentração maior de cárie dentária foi comprovada em um terço dos estudantes avaliados. Estes resultados corroboram os trabalhos similares, como os realizados em Barcelos (AM) ceo-d 2,96 (SiC Index 6,39) para as idades de 6 a 11 anos e CPO-D de 1,89 (SiC Index 4,22) aos de 12 a 17 anos. ${ }^{28}$ Bem como, encontrado em Belo Horizonte (Brasil) ceo-d 1,48 (SiC Index 3,37) e CPO-D 0,57 ( $\mathrm{SiC}$ Index 2,20) e em Córdoba (Argentina) ceo-d 2,38 (SiC Index 5,56) e CPO-D 0,81 ( $\mathrm{SiC}$ Index 2,17), na faixa etária de 6 a 12 anos. ${ }^{15}$ Assim como, no estudo realizado no estado de São Paulo (SP) CPO-D 2,94 ( $\mathrm{SiC}$ Index 5,81) em cidades que possuem flúor nas águas de abastecimento público e CPO-D 3,89 (SiC Index 7,19) em cidades que não possuem flúor nas águas de abastecimento, nas idades entre 11 e 12 anos. ${ }^{16}$ Como também, em Indaiatuba (SP) CPO-D 2,50 (SiC index 5,97). ${ }^{29}$ Evidenciando neste estudo uma distribuição desarmônica da cárie dentária e reforçando a importância em se planejar estratégias adequadas voltadas para esse grupo com maior concentração da doença, objetivando diminuir a prevalência e a severidade da cárie dentária. ${ }^{10,12}$

A variável sexo mostrou significância em relação ao desfecho estudado, divergindo dos resultados encontrados em outros estudos. ${ }^{4,10,25,28,29}$ Entretanto, sabe-se que frequentemente 0 irrompimento dos dentes ocorre, primeiro no sexo feminino e, dependendo da idade analisada e/ou nível de experiência de cárie, pode-se encontrar diferenças quanto ao gênero em função do risco relacionado ao tempo de exposição dos dentes. ${ }^{30}$

Destaca-se que, nesse estudo, a necessidade de tratamento restaurador é a mais evidente. Isso pode ser explicado devido a uma elevada demanda reprimida no município sem acesso a ABS, corroborando os resultados encontrados em crianças e adolescentes de 2 a 13 anos em Camaragibe (PE), ${ }^{31}$ nos escolares de doze anos em Indaiatuba (SP) ${ }^{29}$ e também nos escolares de 6 a 11 anos e de 12 a 17 anos em Barcelos (AM), evidenciando a necessidade de um tratamento odontológico mais conservador. ${ }^{12}$ Entretanto, foi encontrado na dentição permanente um percentual significativo de adolescentes com indicação de tratamento pulpar e extração, sugerindo que esses dentes deixaram de ser restaurados em determinado momento e agora necessitam de tratamento menos conservador, ${ }^{12}$ o que amplia a ocorrência de edentulismo na idade adulta e idosa desses jovens. Nesse sentido, recomenda-se a ampliação da cobertura da ESF do município e o fortalecimento do trabalho interprofissional das equipes de saúde, contribuindo tanto para a prevenção da ocorrência da doença nas crianças e adolescentes, como também minimizando as sequelas produzidas e pelas necessidades de tratamentos invasivos. Até o ano de 2017, a sede do município contava com apenas três ESB inseridas na ESF. Além do mais, embora o município não possua o benefício da fluoretação da água de abastecimento público, essa tecnologia de saúde pública utilizada há mais de meio século no Brasil deve ser implementada, de modo a reduzir a carga de doença sobre o pólo mais atingido, em decorrência de sua comprovada eficácia preventiva. ${ }^{26,27,32}$

Um estudo realizado nas ESB em todo o território nacional apontou que $45,1 \%$ da população brasileira têm dificuldades de acesso à consulta odontológica, 34,5\% não conseguem agendar atendimento e $20,4 \%$ nunca procuraram. O estudo informou ainda que a organização da demanda é um dos problemas encontrados nos serviços públicos de saúde, uma vez que são direcionados para o atendimento por livre demanda, cujos principais problemas são prática assistencial mutiladora e aqueles serviços em que os atendimentos só ocorrem de forma programada, quando a restrição ao acesso é o principal problema. ${ }^{33} \mathrm{Um}$ dos grandes desafios da gestão setorial é produzir mecanismos que induzam à reorganização do processo de trabalho e criar formas participativas e integradas de porta de entrada que ampliem o acesso, garantindo a integralidade, o cuidado humanizado e a promoção da saúde. ${ }^{33,34}$

A triagem visual é uma das ferramentas mais importantes para a prevenção da cegueira. ${ }^{5}$ Quanto maior o atraso na detecção das deficiências visuais, menores são as chances de recuperação e correção do problema, uma vez que pode ser corrigida com terapêutica adequada. ${ }^{35,36}$ Portanto, a implementação dos programas relacionados à detecção precoce de problemas de visão, além de ser menos oneroso do que aqueles representados pelo atendimento a portadores de distúrbios oculares, contribui para uma população mais saudável. ${ }^{9,35,37}$

Segundo dados do censo do Instituto Brasileiro de Geografia e Estatística (IBGE), ${ }^{21}$ em 2010, 23,9\% da população brasileira são portadores de deficiência visual, seja ela total ou parcial. Os dados encontrados nesse estudo $(8,4 \%)$ são condizentes com os trabalhos de Régis-Aranha et al. ${ }^{38} 6,3 \%$; Mello et al. ${ }^{9} 12,8 \%$; Granzoto et al. ${ }^{35} 15,1 \%$; Becker et al. ${ }^{36} 17,4 \%$; Lucena et al. ${ }^{37}$ $29,1 \%$ e de Schumaher e Gasparetto ${ }^{39} 26,6 \%$. Trabalhos científicos mostram que a prevalência de baixa acuidade visual varia de 3,5 a $34,8 \%,{ }^{36}$ logo o presente trabalho se encontra dentro da média esperada.

Quando foi comparada a baixa acuidade visual com o tipo de escola, o resultado do presente estudo corrobora os trabalhos realizados no município de Onça de Pitangui, em Minas Gerais, onde $30,2 \%$ das crianças e adolescentes das escolas estaduais foram diagnosticadas com alterações na acuidade visual. ${ }^{40}$

Tendo em vista que alguns estudos vêm discutindo a deficiência visual e a relação com a odontologia, ${ }^{19,28}$ o presente estudo não evidenciou associação entre déficit visual e a ocorrência de cárie dentária. São necessários estudos futuros envolvendo amostra com outras idades para melhor compreender a relação entre cárie dentária e baixa acuidade visual.

Esse estudo teve como limitações: ter sido realizado do tipo transversal, com amostra de conveniência e por ser conduzido apenas na área urbana de Barreirinha, o que talvez possa não corresponder à realidade do município como um todo.

Apesar das limitações, o presente estudo é relevante por ser o primeiro a colaborar para um diagnóstico inicial da 
saúde bucal e saúde visual desses escolares pesquisados, como também poderá fornecer subsídios para o planejamento de ações educativas, preventivas e curativas direcionadas às necessidades em saúde desses jovens, visando à redução dos impactos negativos que podem acarretar, contribuindo também para que as equipes ofertem cada vez mais um cuidado integral à saúde bucal da população e não apenas sanar as sequelas produzidas pelas doenças.

\section{CONCLUSÃO}

A partir dos resultados deste estudo, uma parcela significativa de escolares apresentou elevada prevalência de cárie dentária, como também encontrou associação entre esta doença e sexo. Evidenciou-se também uma baixa prevalência de dificuldades visuais entre os alunos matriculados no ensino fundamental da rede pública de ensino de Barreirinha, município do estado do Amazonas.

\section{AGRADECIMENTOS}

O presente trabalho foi realizado com o apoio da Escola Superior de Ciências da Saúde, Universidade do Estado do Amazonas (ESA/UEA) e pela Faculdade de Odontologia de Piracicaba FOP/UNICAMP.

\section{CONTRIBUIÇÕES DOS AUTORES}

Desenho do estudo. Lauramaris de Arruda Regis-Aranha. Marcelo de Castro Meneghim. Ângelo Esmael da Silva Maklouf. Guilherme Regis Aranha. Adriana Beatriz Silveira Pinto.

Coleta de dados. Lauramaris de Arruda Regis-Aranha. Shirley Maria de Araújo Passos.

Análise de dados. Lauramaris de Arruda Regis-Aranha. Marcelo de Castro Meneghim. Adriana Beatriz Silveira Pinto.

Interpretação dos resultados. Lauramaris de Arruda RegisAranha. Marcelo de Castro Meneghim. Ângelo Esmael da Silva Maklouf. Guilherme Regis Aranha. Adriana Beatriz Silveira Pinto. Shirley Maria de Araújo Passos.

Redação e revisão crítica do manuscrito. Lauramaris de Arruda Regis-Aranha. Marcelo de Castro Meneghim. Ângelo Esmael da Silva Maklouf. Guilherme Regis Aranha. Adriana Beatriz Silveira Pinto. Shirley Maria de Araújo Passos. Ângela Xavier Monteiro.

Aprovação da versão final do artigo. Lauramaris de Arruda Regis-Aranha. Marcelo de Castro Meneghim. Ângelo Esmael da Silva Maklouf. Guilherme Regis Aranha. Adriana Beatriz Silveira Pinto. Shirley Maria de Araújo Passos. Ângela Xavier Monteiro.

Responsabilidade por todos os aspectos do conteúdo e a integridade do artigo publicado. Lauramaris de Arruda RegisAranha. Marcelo de Castro Meneghim. Ângelo Esmael da Silva Maklouf. Guilherme Regis Aranha. Adriana Beatriz Silveira Pinto. Shirley Maria de Araújo Passos. Ângela Xavier Monteiro.

\section{EDITOR ASSOCIADO}

Aline Cristiane Okido Cavicchioli

\section{REFERÊNCIAS}

1. Ministério da Saúde (BR), Secretaria de Atenção Básica, Departamento de Atenção Básica. Cadernos de Atenção Básica - Saúde na Escola [Internet]. Brasília; 2009 [citado 2020 Mar 19]. Disponível em: http://189.28.128.100/dab/docs/publicacoes/cadernos_ab/abcad24. pdf

2. Ministério da Saúde (BR). Política Nacional de Atenção Integral à Saúde da Criança: orientações para implementação [Internet]. Brasília; 2018 [citado 2020 Ago 24]. Disponível em: https://portaldeboaspraticas.iff. fiocruz.br/wp-content/uploads/2018/07/Pol\%C3\%ADtica-Nacionalde-Aten\%C3\%A7\%C3\%A3o-Integral-\%C3\%A0-Sa\%C3\%BAde-daCrian\%C3\%A7a-PNAISC-Vers\%C3\%A3o-Eletr\%C3\%B4nica.pdf

3. Garcia LC, Uchida TH, Lima JPG, Terada RSS, Pascotto RC, Fujimaki M Práticas de educação em saúde para a prevenção da cárie dentária: um estudo qualitativo com cirurgiões-dentistas. Rev ABENO. 2018;18(3):6274. http://dx.doi.org/10.30979/rev.abeno.v18i3.498.

4. Barros WRC, Nascimento LS, Fontes RBC, Aguiar NL, Silva Jr IF, Souza CNP. Prevalência de cárie dentária na adolescência em Belém do Pará: uma perspectiva Amazônica. Adolesc Saude [Internet]. 2015; [citado 2020 Jul 1];1(2):59-68. Disponível em: https://cdn.publisher.gn1.link/ adolescenciaesaude.com/pdf/v12n2a07.pdf

5. Pereira CFA, Costa R, Ciampo LAD, Ferraz I. Triagem de acuidade visual reduzida em uma unidade de Atenção Primaria a Saúde. Rev Bras Oftalmol. 2019;78(4):250-4. http://dx.doi.org/10.5935/00347280.20190138.

6. Peres MA, Macpherson LMD, Weyant RJ, Daly B, Venturelli R, Mathur MR et al. Oral diseases: a global public health challenge. Lancet 2019;394(10194):249-60. http://dx.doi.org/10.1016/S0140-6736(19)311468.

7. Ministério da Saúde (BR), Secretaria de Atenção à Saúde, Secretaria de Vigilância em Saúde. SB Brasil 2010: pesquisa nacional de saúde bucal: resultados principais [Internet]. Brasília; 2012 [citado 2020 Mar 19]. Disponível em: http://bvsms.saude.gov.br/bvs/publicacoes/ pesquisa_nacional_saude_bucal.pdf

8. Freire MCM, Reis SCGB, Figueiredo N, Peres KG, Moreira RS, Antunes JLF. Determinantes individuais e contextuais da cárie em crianças brasileiras de 12 anos em 2010. Rev Saude Publica. 2013;47(3, Supl. 3):40-9. http://dx.doi.org/10.1590/S0034-8910.2013047004322.

9. Mello MAFC, Rodrigues RM, Conterno SFR, Toso BRGO, Missio L. Avaliações de saúde de escolares no programa saúde na escola. Rev Interdisciplin Estud Saúde [Internet]. 2019; [citado 2020 Jul 1];9(2):261. 77. Disponível em: https://periodicos.uniarp.edu.br/index.php/ries/ article/view/1546/1083

10. Brito ACM, Bezerra IM, Cavalcante DFB, Pereira AC, Vieira V, Montezuma MF et al. Dental caries experience and associated factors in 12-yearold-children: a population based-study. Braz Oral Res. 2020;34:e010 http://dx.doi.org/10.1590/1807-3107bor-2020.vol34.0010.

11. Amaral RC, Fonseca EP, Lepri CP, Assis LC, Rocha CM, Tennant M. Cárie dentária em adolescentes do Estado de São Paulo, Brasil: uma análise espacial, 2015. Adolesc Saude [Internet]. 2019; [citado 2020 Jul 1];16(4):25-35. Disponível em: https://cdn.publisher.gn1.link/ adolescenciaesaude.com/pdf/v16n4a04.pdf

12. Sousa MLR, Rando-Meirelles MPM, Tôrres LHN, Frias AC. Cárie dentária e necessidade de tratamento em adolescentes paulistas. Rev Saude Publica. 2013;47(Supl. 3):50-8. http://dx.doi.org/10.1590/ S0034-8910.2013047004340.

13. Demeu AJM, Viudes LP, Barbosa LC, Nascimento VR. Prevalência de cárie em crianças do ensino fundamental de Umuarama, Paraná. Arch Health Invest. 2019;8(10):592-6. http://dx.doi.org/10.21270/archi. v8i10.3820.

14. Cardoso L, Rosing C, Kramer P, Costa CC, Costa Fo LC. Polarização da cárie em município sem água fluoretada. Cad Saude Publica. 2003;19(1):237-43. http://dx.doi.org/10.1590/S0102-311X2003000100026.

15. Gomes VE, Ferreira RC, Morais MAS, Houri LCLF, Bella MI, Cornejo LS et al. Cárie dentária na América do Sul: realidade entre escolares do Brasil e Argentina. J. Health Biol Sci. 2019;7(2):152-8. http://dx.doi. org/10.12662/2317-3076jhbs.v7i2.2570.p152-158.2019. 
16. Antunes JLF, Narvai PC, Nugent ZJ. Measuring inequalities in the distribution of dental caries. Community Dent Oral Epidemiol. 2004;32(1):41-8. http://dx.doi.org/10.1111/j.1600-0528.2004.00125.x.

17. Ministério da Saúde (BR). Projeto Olhar Brasil: triagem de acuidade visual: manual de orientação [Internet]. Brasília; 2008 [citado 2020 Ago 24]. Disponível em: http://portal.mec.gov.br/index.php?option=com docman\&view=download\&alias=1863-pse-manual-olharbrasil\&ltemid=30192

18. Amaral A, Melão N. O perfil de saúde de crianças vigiadas em consultas de cuidados primários na cidade de Viseu, Portugal. Rev Port Saude Publica. 2016;34(1):53-60. http://dx.doi.org/10.1016/j.rpsp.2015.07.003.

19. Monteiro LPA, Pereira RM, Monteiro ACC, Costa ICC. O conhecimento de deficientes visuais em relação à saúde bucal. Rev Ciênc Plur [Internet]. 2018; [citado 2020 Jul 1];4(1):44-66. Disponível em: https://periodicos. ufrn.br/rcp/article/view/14476/9820

20. Secretaria Nacional de Promoção dos Direitos da Pessoa com Deficiência, Coordenação Geral do Sistema de Informações sobre a Pessoa com Deficiência. Cartilha do censo 2010 pessoa com deficiência [Internet]. Brasília: Secretaria de Direitos Humanos;2012. p. 32 [citado 2020 Mar 19]. Disponível em: https://docplayer.com.br/239748-Cartilha-do-censo2010-pessoas-com-deficiencia.html

21. Instituto Brasileiro de Geografia e Estatística. Censo demográfico 2010 [Internet]. Rio de Janeiro: IBGE;2010 [citado 2020 Mar 19]. Disponível em: https://www.ibge.gov.br/pt/inicio.html

22. Ministério da Saúde. Informação e Gestão da Atenção Básica. Relatórios públicos dos Sistemas da Atenção Básica [Internet]. Brasília: Cobertura da Saúde Bucal; 2017 [citado 2020 Mar 19]. Disponível em: https:// egestorab.saude.gov.br/

23. Programa das Nações Unidas para o Desenvolvimento. Brasil: Ranking IDHM Municípios [Internet]. 2010 [citado 2020 Mar 19]. Disponível em: https://www.br.undp.org/content/brazil/pt/home/idho/rankings/idhmmunicipios-2010.html

24. World Healt Organization. Oral health surveys: basic methods. Geneva: WHO; 2013.

25. Frazão P, Santos CRI, Benicio DEDA, Marques RAA, Benício MHDA, Cardoso MA et al. Cárie dentária em escolares de 12 anos de idade em município sem água fluoretada na Amazônia Ocidental brasileira, 2010. Epidemiol Serv Saude. 2016;25(1):149-58. http://dx.doi.org/10.5123/ S1679-49742016000100015.

26. Narvai PC, Frias AC, Fratucci MVB, Antunes JLF, Carnut L, Frazão P. Fluoretação da água em capitais brasileiras no início do século XXI: a efetividade em questão. Saúde Debate. 2014;38(102):562-71. http:// dx.doi.org/10.5935/0103-1104.20140052.

27. Cruz MGB, Narvai PC. Cárie e água fluoretada em dois municípios brasileiros com baixa prevalência da doença. Rev Saude Publica. 2018;52:28. http://dx.doi.org/10.11606/S1518-8787.2018052016330.

28. Régis-Aranha LA, Santos STC, Magalhães WOG, Pinto ABS, Passos SMA, Monteiro AX. Dental caries and visual acuity of students in a town in west Amazon. Braz J Oral Sci. 2018;17:e18159. http://dx.doi. org/10.20396/bjos.v17i0.8653816.
29. Rihs LB, Sousa MLR, Cypriano S, Abdalla NM. Desigualdades na distribuição da cárie dentária em adolescentes de Indaiatuba (SP) 2004. Cien Saude Colet. 2010;15(4):2173-80. http://dx.doi.org/10.1590/ S1413-81232010000400031.

30. Cypriano S, Hugo NF, Sciamarelli MC, Tôrres LHN, Sousa MLR, Wada RS. Fatores associados à experiência de cárie em escolares de um município com baixa prevalência de cárie dentária. Cien Saude Colet. 2011;16(10):4095-106. http://dx.doi.org/10.1590/S141381232011001100015.

31. Souza EHA, Oliveira PAP, Galvão PVM. Necessidade de tratamento odontológico em crianças do município de Camaragibe na faixa etária de 2 a 13 anos. Odontol. Clín. Cient [Internet]. 2012; [citado $2020 \mathrm{Jul}$ 1];11(3):215-9. Disponível em: http://revodonto.bvsalud.org/pdf/occ/ v11n3/a08v11n3.pdf

32. Peres MA, Liu P, Demarco FF, Silva AER, Wehrmeister FC, Menezes $\mathrm{AM}$ et al. Income trajectories affect treatment of dental caries from childhood to young adulthood: a birth cohort study. Braz Oral Res. 2018;32(0):e36. http://dx.doi.org/10.1590/1807-3107bor-2018.vol32.0036.

33. Casotti E, Contarato PC, Fonseca ABM, Borges PKDO, Baldani MH Atenção em saúde bucal no Brasil: uma análise a partir da avaliação externa do PMAQ-AB. Saúde Debate. 2014;38(special):140-57. http:// dx.doi.org/10.5935/0103-1104.2014S011.

34. Neves M, Giordani JMA, Hugo FN. Atenção primária à saúde bucal no Brasil: processo de trabalho das equipes de saúde bucal. Cien Saude Colet. 2019;24(5):1809-20. http://dx.doi.org/10.1590/141381232018245.08892017

35. Granzoto JA, Ostermann CSPE, Brum LF, Pereira PG, Granzoto T. Avaliação da acuidade visual em escolares da 1a série do ensino fundamental. Arq Bras Oftalmol. 2003;66(2):167-71. http://dx.doi. org/10.1590/S0004-27492003000200010.

36. Becker TOF, Cortela DCB, Miura H, Matsuhara ML. Evaluation of visual acuity in municipal elementary school students. Rev Bras Oftalmol. 2019;78(1):37-41. http://dx.doi.org/10.5935/0034-7280.20190008.

37. Lucena BM, Machado LA, Barreto PMS, Tavares PM, Rodrigues AMH Solari HP et al. Prevalência de fatores predisponentes de baixa visual em uma população de jovens do Colégio Universitário Geraldo Reis em Niterói - RJ. Rev Bras Oftalmol. 2019;78(6):380-3. http://dx.doi. org/10.5935/0034-7280.20190165.

38. Régis-Aranha LA, Moraes FH, Santos STC, Heufemann NEC, Magalhães WOG, Zacarias Fo RP et al. Visual acuity and academic performance of students in a Brazilian Amazon municipality. Esc Anna Nery Rev Enferm. 2017;21(2):e20170032. http://dx.doi.org/10.5935/1414-8145.20170032.

39. Schumaher MLN, Gasparetto MERF. Saúde ocular de escolares: atuação de enfermeiros. Rev Enferm UERJ [Internet]. 2016; [citado $2020 \mathrm{Jul}$ 1];24(6):e15108. Disponível em: https://www.e-publicacoes. uerj.br/index.php/enfermagemuerj/article/view/15108/20984

40. Procópio MEN, Takahashi DM, De-Souza GFR. Avaliação da acuidade visual em escolares e adolescentes na cidade de Onça de Pitangui, Minas Gerais, Brasil. Rev Méd Minas Gerais. 2019;29:e-2033. http:// dx.doi.org/10.5935/2238-3182.20190051. 